\title{
How do we maintain competence in aneurysm surgery
}

\author{
Bruno M. Santiago • Manuel Cunha e Sá
}

Received: 19 October 2014 / Accepted: 22 October 2014 / Published online: 14 November 2014

(C) Springer-Verlag Wien 2014

The article by Le Reste et al. [7] addresses a very important topic in our current cerebrovascular practice. In order to deliver to our patients the best quality of care, a certain level of proficiency and competence needs to be attained and maintained. This is true for all areas of neurosurgery and constitutes a realistic objective in the treatment of most pathologies.

The authors have looked at a particular subset of patients for whom surgery remains the recommended form of treatment. In fact, in high-grade SAH patients with intracerebral space-occupying hematomas, surgical decompression of the mass effect may be warranted, and along with it the clipping of the bleeding aneurysm. The authors suggest a correlation between cumulative surgical experience and lower intraoperative aneurysm rupture, although mortality and final outcome did not reach statistical significance. This can in fact be due to the sample size and the lower number of cases treated by surgeons with less cumulative experience. Five surgeons operated on these difficult patients on an emergency basis, one of them with minimal experience in the elective surgical treatment of aneurysms (surgeon 1 with only ten cumulative cases, nine of them within the study). There is no reference as to who was assisting these surgeons and what kind of experience these assistants had.

Of course the surgical scenario in such challenging cases is far from being favorable. The brain is angry and edematous, intracranial pressure is flying high, not always with dilated ventricles enabling ventricular drainage, arachnoid spaces are collapsed with opercula overlying each other, which makes the dissection significantly more difficult than usual because the brain and veins become rather more friable. The surgeon has to constantly worry, control, and override all these

B. M. Santiago · M. Cunha e Sá $(\bowtie)$

Department of Neurosurgery, Hospital Garcia de Orta EPE, Av.

Torrado da Silva, 2801-951 Almada, Portugal

e-mail: mcunhaesa@gmail.com difficulties, draining the hematoma, securing space and surgical conditions, which will enable him to control the circulation and deal with the ruptured aneurysm. In emergency cases like these, there is little time and room for the usual discussion regarding the size and shape of the sac and neck, the existence of calcified walls, and whether there are arteries taking off directly from the sac. In other words, all considerations that are pertinent in the context of an elective choice between a surgical or an endovascular treatment are cast away by the need to surgically decompress the hematoma, secure the aneurysm, and control ICP. As wisely pointed out by the authors, the surgeon needs to be prepared to circumvent all these difficulties, having as background the particularly adverse scenario of an urgent or emergent procedure in an unstable patient with poor medical condition. Both very important technical and also non-technical factors are at play. The technical gesture will only be perfect if the psychological condition of the surgeon and his resilience to act in severe adversity exists. The conjunction of the former and latter factors will only occur if the acting surgeon has acquired and maintained a level of competence, which will enable him to rise to the circumstances. This objective can only be secured if a significant enough number of aneurysms remain in the hands of neurosurgeons to treat.

As stated by the authors, this matter has been the subject of a number of publications in our literature addressing the issues involved in overcoming the learning curves existing for the treatment of several neurosurgical entities.

Gathering from the surgical literature as a whole, both individual surgeon performance and hospital volume have been correlated with better outcomes for a variety of surgical procedures, meaning that the global performance of the system of care is essential for achieving the goal of the best possible outcome [4]. In the particular case of aneurysm surgery, as well as in other areas where strong dispute is at play with other therapeutic modalities, which have proved 
successful in the treatment of the same disease, teaching and training of the surgical gestures is certainly at risk. Not only the number (quantity) but also the diversity (quality) of cases may become so limited as to endanger, frustrate, or even make it impossible to secure the objective of mastering the surgical technique required in the surgical treatment of aneurysms. Adaptation to this new reality has prompted technology and imagination with new solutions, such as the development of software applications, surgical simulators, and even prototype models of diseased vessels [6]. These alternatives tend to recreate the problems and gestures one is usually faced with when performing a real surgery, allowing for some part of the necessary training to be acquired on a dummy model and not at the cost of the patient, thereby shortening the surgical caseload learning curve.

Unfortunately, these techniques are still at an early stage of development and their application remains quite variable depending on the type or surgical act $[2,6]$.

Certainly, the same training contingencies exist for other treatment modalities, endovascular included, but the issue becomes even hotter for the surgical treatment of aneurysms, as numbers seem to decline to levels incompatible with training and competence.

The issue could easily be solved on its own if a clear, sustained, and significant superiority of the endovascular technique would be consistently present compared with the surgical treatment. Several publications addressing this important issue in the recent past seem not to sustain such assertion $[1,9,10]$. A significant number of aneurysms are still treated with surgical clipping in a more efficacious and long-lasting manner. On a paper presented at the AANS meeting earlier this year [11], Spetzler et al. discussed the 6-year results of the BRAT study. A case was made for surgical treatment for anterior circulation aneurysms, given the similar outcomes, higher obliteration rate ( 98 vs. $48 \%$ ), lower retreatment rate (4 vs. $13 \%$ ), and high crossover from the coil to clip arm (38\%). For posterior circulation aneurysms, outcomes were definitely better for endovascular [11].

Furthermore, recent technological advances in endovascular techniques, which seem promising for an increasing number of complex aneurysms, carry an increased morbidity rate that must be factored in when treatment decisions are made, such as with the use of stents in ruptured aneurysms [8]. The decision-making process and patient selection for ruptured aneurysms treatment has evolved more as an art than as a science [3], which is a consequence of the collaborative efforts occurring between all subspecialties involved, the availability of both techniques on a $24-48-\mathrm{h}$ window of treatment, the anatomical factors of the aneurysm neck and sac, as well as local expertise and morbidity factors involved with both options of treatment. Specific subsets of aneurysms are more favorably treated by one particular technique, emphasizing the need for the simultaneous availability of microsurgery and endovascular treatment operating at a high quality level in cerebrovascular centers treating these patients. This reality may meet with different ends depending on countries, regions, and hospitals. In Europe, where a trend towards a dichotomous view of exclusive and opposing options for securing aneurysms can sometimes be perceived [3], the problem of maintaining competence in aneurysm surgery has become more acute.

Neurosurgery must keep its leadership role in cerebrovascular surgery by assuring microsurgical proficiency of a younger generation of surgeons embracing the treatment of cerebrovascular disorders, but also by developing a consistent endovascular expertise. In the US, a number of training programs are including endovascular exposure to residents during their training, assuming the endovascular suite as a regular OR room $[5,9]$. The training of surgeons in both techniques seems promising and the right way to go, regardless of whether a dually trained neurosurgeon will end up opting for the use of one technique over the other. The important is that we guarantee the ability to deliver our patients the best possible care by providing them with a choice that is not born out of a turf war but based on evidence both on a general, but similarly important, local one.

Conflicts of interest None.

\section{References}

1. Bakker NA, Metzemaekers JD, Groen RJ, Mooij JJ, Van Dijk JM (2009) International subarachnoid aneurysm trial 2009: endovascular coiling of ruptured intracranial aneurysms has no significant advantage over neurosurgical clipping. Neurosurg 66(5):961-962

2. Chan S, Conti F, Salisbury K, Blevins NH (2013) Virtual reality simulation in neurosurgery: technologies and evolution. Neurosurg 72:154-164

3. Chowdhry S, Spetzler RF (2014) Genealogy of training in vascular neurosurgery. Neurosurg 74(Suppl 2):S198-203

4. Gawande A (2001) Creating the educated surgeon in the 21 st century. Am J Surg 181(6):551-556

5. Harbaugh RE, Agarwal A (2006) Training residents in endovascular neurosurgery. Neurosurg 59(5 Suppl 3):S277-281

6. Kimura T, Morita A, Nishimura K, Aiyama H, Itoh H, Fukaya S, Sora S, Ochiai C (2009) Simulation of and training for cerebral aneurysm clipping with 3-dimensional models. Neurosurgery 65:719-726

7. Le Reste PJ, Henaux PL, Riffaud L, Haegelen C, Morandi X (2014) Influence of cumulative surgical experience on the outcome of poorgrade patients with ruptured intracranial aneurysm. Acta Neurochir (Wien) Sep 25 [Epub ahead of print]

8. Piotin M, Blanc R, Spelle L, Mounayer C, Piantino R, Schmidt PJ, Moret J (2010) Stent-assisted coiling of intracranial aneurysms: clinical and angiographic results in 216 consecutive aneurysms. Stroke 41(1):110-115

9. Sauvageau E, Hopkins LN (2006) Training in cerebrovascular disease: do we need to change the way we train residents. Neurosurg 59(5 Suppl 3):S282-286 
10. Spetzler RF, McDougall CG, Albuquerque FC, Zabramski JM, Hills NK, Partovi S, Nakaji P, Wallace RC (2013) The Barrow Ruptured Aneurysm Trial: 3-year results. J Neurosurg 119: $146-157$
11. Spetzler RF, McDougall CG, Albuquerque FC, Zabramski JM, Hills NK, Partovi S, Nakaji P, Wallace RC (2014) The Barrow Ruptured Aneurysm Trial: 6-year results. Paper presented at 82nd AANS Annual Scientific Meeting: April 5-9 2014; San Francisco, CA 